\title{
Incidência e Preditores Contemporâneos de Complicações Vasculares após Intervenção Coronária Percutânea
}

\author{
Fábio S. Brito Jr. ${ }^{1}$, Marco Aurélio de Magalhães ${ }^{1}$, Teresa Cristina D. C. Nascimento ${ }^{1}$, \\ Ivanise M. G. Amorim', Breno O. Almeida', Alexandre Abizaid', Marco A. Perin'
}

\section{RESUMO}

Fundamentos: A despeito dos avanços da Cardiologia Intervencionista, as complicações vasculares permanecem relativamente freqüentes e seus preditores, na prática médica atual, são pouco conhecidos. A presente investigação foi idealizada com o objetivo de avaliar a incidência e os preditores contemporâneos de complicações vasculares após intervenções coronárias realizadas por via percutânea femoral. Método: Entre dezembro de 2005 e dezembro de 2006, 383 pacientes consecutivos foram submetidos a intervenção coronária percutânea por via femoral e foram monitorizados clinicamente, durante a internação hospitalar, para a detecção de complicações vasculares. Resultados: Complicações vasculares ocorreram em $6,5 \%$ dos pacientes, sendo o pseudo-aneurisma da artéria femoral a complicação mais freqüentemente observada $(2,6 \%)$. Os preditores independentes de complicação vascular, neste estudo, foram o sexo feminino (odds ratio $[\mathrm{OR}]=5,61$; intervalo de confiança de 95\% [IC 95\%] = 1,99-15,76) e o uso do abciximab (OR $=3,02 ;$ IC $95 \%=1,10-8,26)$. O emprego de dispositivos hemostáticos $(O R=0,36$; IC 95\% = 0,12$0,99)$ foi o único fator protetor independente contra a ocorrência dessas complicações, especialmente no sexo masculino. Conclusões: As complicações vasculares ainda são relativamente freqüentes após intervenções coronárias percutâneas. Os preditores contemporâneos dessas complicações são o sexo feminino e o uso de abciximab. O uso de dispositivos hemostáticos, especialmente em indivíduos do sexo masculino, é um fator protetor independente contra a ocorrência dessas complicações.

DESCRITORES: Angioplastia transluminal percutânea coronária, efeitos adversos. Fatores de risco. Doenças vasculares, etiologia. Cateterismo cardíaco, efeitos adversos. Vasos sanguíneos, lesões.

\footnotetext{
1 Hospital Israelita Albert Einstein - São Paulo, SP.

Correspondência: Fábio Sândoli de Brito Jr. Rua Dom Armando Lombardi, 819/82-A - São Paulo, SP - CEP 05616-011 - Tel.: (11) 3747-0434 - Fax: (11)3747-1035 • E-mail: fsbrito@einstein.br Recebido em: 20/9/2007 - Aceito em: 15/10/2007
}

\section{SUMMARY}

\author{
Incidence and Contemporary Predictors of \\ Vascular Complications after Percutaneous \\ Coronary Interventions
}

Background: Despite all advances in interventional cardiology, vascular complications remain relatively frequent and its contemporary predictors are not well studied. The current investigation was designed with the aim of evaluating the incidence and predictors of vascular complications after percutaneous coronary interventions performed by the femoral approach. Method: Between December 2005 and December 2006, 383 consecutive patients were submitted to percutaneous coronary interventions using the femoral approach and were clinically monitored for the detection of in-hospital vascular complications. Results: Vascular complications occurred in $6.5 \%$ of the patients and pseudoaneurism was the most frequently observed complication $(2.6 \%)$. The independent predictors of vascular complications in the present study were female gender (odds ratio $=5.61 ; 95 \% \mathrm{Cl}=1.99-15.76$ ) and the use of abciximab (odds ratio $=3.02 ; 95 \% \mathrm{Cl}=1.10-8.26$ ). Vascular closure devices were the only independent protectors against vascular complications (odds ratio $=0.36$; $95 \% \mathrm{Cl}=0.12-0.99)$, specially when used in men. Conclusion: Vascular complications remain relativelly frequent after percutaneous coronary interventions. The contemporary predictors of these complications are female gender and the use of abciximab, while vascular closure devices act as independent protector against them, specially in men.

DESCRIPTORS: Angioplasty, transluminal, percutaneous coronary, adverse effects. Risk factors. Vascular diseases, etiology. Heart catheterization, adverse effects. Blood vessels, injuries. 
Brito Jr. FS, et al. Incidência e Preditores Contemporâneos de Complicações Vasculares após Intervenção Coronária Percutânea. Rev Bras Cardiol Invas 2007; 15(4): 394-399.

relacionados à intervenção, além de causarem grande desconforto aos pacientes e se relacionarem a maiores taxas de eventos adversos a longo prazo ${ }^{1,5-8}$. Nos últimos anos, observaram-se diversos avanços na Cardiologia Intervencionista. O impacto desses avanços sobre a incidência e os preditores de complicações vasculares foi pouco estudado. A miniaturização dos dispositivos, a redução expressiva da utilização dos aterótomos e a melhoria da técnica podem ter contribuído favoravelmente para a menor ocorrência dessas complicações. Por outro lado, a realização de procedimentos mais complexos e o regime de antiagregação plaquetária mais potente podem ter tido efeito contrário, desfavorável. Adicionalmente, os dispositivos hemostáticos foram incorporados à prática clínica e seu impacto sobre a ocorrência de complicações vasculares permanece controverso ${ }^{4,9-14}$. Pelo exposto acima, decidimos realizar a presente investigação prospectiva, objetivando avaliar a incidência e os preditores contemporâneos de complicações vasculares após intervenções coronárias realizadas por via percutânea femoral.

\section{MÉTODO}

\section{População do estudo}

Entre dezembro de 2005 e dezembro de 2006, 383 pacientes consecutivos foram submetidos a intervenção coronária percutânea por via femoral e foram monitorizados clinicamente, durante a internação hospitalar, para a detecção de complicações vasculares.

\section{Intervenção coronária percutânea}

As intervenções coronárias foram realizadas utilizando-se técnicas convencionais. Os pacientes receberam aspirina (200 mg/dia) e clopidogrel (dose de ataque de $300 \mathrm{mg}$ ou $600 \mathrm{mg}$, seguida de $75 \mathrm{mg} / \mathrm{dia}$ ). O nível de anticoagulação foi monitorizado durante todas as intervenções, utilizando-se o tempo de coagulação ativada (TCA) medido pelo equipamento Hemocron 801. Os pacientes deste estudo receberam, no mínimo, $100 \mathrm{U} / \mathrm{kg}$ de heparina não-fracionada no início da intervenção. Doses adicionais desse anticoagulante foram administradas quando necessário, objetivando atingir TCA entre $250 \mathrm{~s}$ e $350 \mathrm{~s}$. Quando se fez uso concomitante de inibidores da glicoproteína IIb/IIla, administrou-se $70 \mathrm{U} / \mathrm{kg}$ de heparina, procurando atingir TCA entre 200 s e 300 s. Os inibidores da glicoproteína Ilb/IIla foram utilizados a critério do operador.

A retirada do introdutor arterial ocorreu imediatamente após a intervenção, quando se empregaram os dispositivos hemostáticos. A decisão sobre a utilização desses dispositivos bem como a escolha do tipo do dispositivo (Angio-Seal ou Perclose) seguiram critérios do operador. Quando a opção foi pela não-utilização dos dispositivos hemostáticos, a retirada do introdutor arterial por compressão manual ocorreu quatro a seis horas após o final da intervenção, com nível de TCA sempre inferior a $180 \mathrm{~s}$.

\section{Complicações vasculares}

Definiu-se como complicação vascular a ocorrência de: 1. pseudo-aneurisma da artéria femoral, 2. hemorragia retroperitoneal, 3. fístula arteriovenosa, 4. trombose ou embolia no leito arterial utilizado como via de acesso, e 5. sangramento externo ou hematoma significativo, quando determinantes de queda dos níveis de hemoglobina superior a $3 \mathrm{~g} / \mathrm{dl}$, necessidade de transfusão de sangue ou prolongamento da internação hospitalar.

\section{Análise estatística}

As variáveis categóricas estão expressas como número e porcentual e as variáveis contínuas como média \pm desvio padrão. Utilizaram-se os testes qui-quadrado ou exato de Fisher, quando apropriados, para comparação de variáveis categóricas, e o teste $t$ não pareado, para comparação de variáveis numéricas contínuas.

Utilizou-se a análise univariada para identificar os fatores relacionados à ocorrência de complicações vasculares. Dentre esses fatores, aqueles com nível de significância $<0,1$ ou relevância clínica foram incluídos no modelo de regressão logística multivariada, objetivando determinar os preditores independentes de complicações vasculares, que se encontram expressos com odds ratio (OR) e intervalo de confiança de 95\% (IC 95\%). Todos os testes foram bicaudados, sendo considerados estatisticamente significantes quando $p \leq 0,05$. Utilizou-se o software SPSS, versão 13.0 (Chicago, Illinois, Estados Unidos).

\section{RESULTADOS}

\section{Dados demográficos e clínicos}

As características demográficas e clínicas dos pacientes estudados encontram-se na Tabela 1. Hipertensão e diabetes foram relativamente freqüentes e a apresentação clínica como síndrome coronária aguda ocorreu em cerca de dois terços dessa população.

\section{Dados do procedimento}

No procedimento, utilizaram-se inibidores da glicoproteína IIb/IIla (abciximab) em 24,8\% dos casos, predominantemente no infarto do miocárdio com supradesnivelamento do segmento ST, situação em que essa medicação foi administrada em 55,4\% dos pacientes. O TCA, em todos os casos, esteve dentro dos limites estabelecidos como ideais. Não existiu diferença nos níveis de TCA, quando comparados os indivíduos dos gêneros masculino e feminino $(324,8 \pm 88$ s vs. 326,7 $\pm 62 \mathrm{~s} ; \mathrm{p}=0,8)$. Utilizaram-se mais freqüentemente introdutores arteriais $6 \mathrm{~F}$ e os dispositivos hemostáticos foram empregados em 56,7\% dos casos. Os dados do procedimento encontram-se detalhados na Tabela 2 .

\section{Complicações vasculares}

As complicações vasculares ocorreram em 6,5\% dos pacientes, e apenas um apresentou mais de uma 
Brito Jr. FS, et al. Incidência e Preditores Contemporâneos de Complicações Vasculares após Intervenção Coronária Percutânea. Rev Bras Cardiol Invas 2007; 15(4): 394-399.

complicação (pseudo-aneurisma e hemorragia retroperitoneal). O pseudo-aneurisma da artéria femoral foi a complicação vascular mais freqüentemente encontrada nessa população, como demonstra a Tabela 3.

As complicações vasculares foram mais freqüentes nas mulheres, em comparação com os homens $(14,8 \%$ vs. $4,3 \% ; p<0,01)$, o que também se observou, numericamente, na análise individualizada de cada tipo de complicação (hematoma, 3,7\% vs. 0,3\%; sangramento externo, 3,7\% vs. 1,7\%; hemorragia retroperitoneal, $2,5 \%$ vs. $0,3 \%$; pseudo-aneurisma, $3,7 \%$ vs. $2,3 \%$; trombose/isquemia, 1,2\% vs. $0 \%$ ).

TABELA 1

Características demográficas e clínicas dos pacientes

\begin{tabular}{lc}
\hline & $\mathbf{n}=\mathbf{3 8 3}$ \\
\hline Idade, anos & $66,4 \pm 12,6$ \\
Sexo masculino & $302(78,9 \%)$ \\
Hipertensão & $254(66,3 \%)$ \\
Diabetes & $122(33,0 \%)$ \\
DMNID & $75(19,6 \%)$ \\
DMID & $47(12,3 \%)$ \\
Tabagismo atual & $72(18,8 \%)$ \\
Dislipidemia & $205(53,5 \%)$ \\
Índice de massa corpórea, kg/m² & $27,3 \pm 4,3$ \\
Quadro clínico & \\
$\quad$ Angina estável/isquemia silenciosa & $141(36,8 \%)$ \\
SCA sem elevação ST & $171(44,5 \%)$ \\
IAM com supra ST & $56(14,6 \%)$ \\
Multiarteriais & $243(63,4 \%)$ \\
\hline $\mathrm{n}=$ número de pacientes; DMNID = diabetes melito não- \\
insulino-dependente; DMID = diabetes melito insulino-de- \\
pendente; SCA = síndrome coronária aguda; IAM = infarto \\
agudo do miocárdio.
\end{tabular}

TABELA 2

Características do procedimento

\begin{tabular}{lc}
\hline & $\mathbf{n}=\mathbf{3 8 3}$ \\
\hline Introdutor & \\
$6 \mathrm{~F}$ & $268(70,0 \%)$ \\
$7 \mathrm{~F}$ & $104(27,2 \%)$ \\
$8 \mathrm{~F}$ & $10(2,6 \%)$ \\
TCA, s & $325 \pm 84,2$ \\
Uso de abciximab & $95(24,8 \%)$ \\
Dispositivo hemostático & $217(56,7 \%)$ \\
Angio-Seal & $200(52,2 \%)$ \\
Perclose & $17(4,4 \%)$ \\
\hline $\mathrm{n}=$ número de pacientes; TCA = tempo de coagulação ativada.
\end{tabular}

\section{Preditores de complicações vasculares}

A análise univariada (Tabela 4) identificou os seguintes fatores relacionados à ocorrência de complicações vasculares: sexo feminino, idade, apresentação clínica como síndrome coronária aguda, e utilização dos inibidores da glicoproteína IIb/IIla. O emprego de dispositivos hemostáticos relacionou-se à menor ocorrência de complicações. Essas variáveis foram forçadas no modelo multivariado de regressão logística, permanecendo o sexo feminino (OR = 5,61; IC 95\% $=1,99-15,76)$ e o uso do abciximab $(\mathrm{OR}=3,02$; IC $95 \%=1,1-8,26)$ como preditores independentes de complicação vascular após intervenção coronária percutânea realizada por via femoral. Nesta mesma análise, o emprego dos dispositivos hemostáticos $(\mathrm{OR}=$ 0,36; IC 95\% = 0,2-0,99) emergiu como o único fator protetor independente contra a ocorrência dessas complicações. Entretanto, ao se analisar separadamente os indivíduos dos gêneros masculino e feminino, observa-se que essa proteção só ocorreu nos homens (complicações com dispositivo $=1,2 \%$ vs. complica-

TABELA 3

Complicações vasculares

\begin{tabular}{lc}
\hline Complicação & n \\
\hline Complicações (por paciente) & $25(6,5 \%)$ \\
Complicações (por evento) & $26(6,8 \%)$ \\
Pseudo-aneurisma & $10(2,6 \%)$ \\
Sangramento externo & $8(2,1 \%)$ \\
Hematoma & $4(1,0 \%)$ \\
Hemorragia retroperitoneal & $3(0,8 \%)$ \\
Isquemia/trombose & $1(0,3 \%)$ \\
\hline
\end{tabular}

TABELA 4

Análise univariada para os preditores de complicação vascular

\begin{tabular}{lccc}
\hline Variável & Odds ratio & IC 95\% & p \\
\hline Sexo feminino & 3,86 & $1,69-8,84$ & 0,001 \\
Idade & 1,03 & $1,00-1,07$ & 0,03 \\
Hipertensão & 1,30 & $0,53-3,20$ & 0,56 \\
Diabetes & 1,00 & $0,39-2,54$ & 1,00 \\
IMC & 0,96 & $0,87-1,05$ & 0,39 \\
Multiarterial & 0,44 & $0,16-1,21$ & 0,11 \\
Introdutor & 0,89 & $0,37-2,14$ & 0,80 \\
Abciximab & 2,14 & $0,92-4,94$ & 0,07 \\
Quadro agudo & 3,12 & $1,04-9,39$ & 0,04 \\
TCA & 0,99 & $0,98-1,00$ & 0,27 \\
Dispositivo hemostático & 0,48 & $0,21-1,09$ & 0,08
\end{tabular}

IC 95\% = intervalo de confiança de 95\%; IMC = índice de massa corpórea; TCA = tempo de coagulação ativada. 
Brito Jr. FS, et al. Incidência e Preditores Contemporâneos de Complicações Vasculares após Intervenção Coronária Percutânea. Rev Bras Cardiol Invas 2007; 15(4): 394-399.

ções sem dispositivo = 8,1\%; $p=0,004 ;$ OR = 0,108; IC $95 \%=0,03-0,47)$. Entre as mulheres, não existiu diferença na ocorrência de complicações, quando comparadas as que utilizaram e as que não utilizaram os dispositivos hemostáticos (15,4\% vs. 13,8\%; $p=0,9)$.

O resultado da análise multivariada encontra-se representado graficamente na Figura 1.

\section{DISCUSSÃO}

Na presente investigação, utilizando técnicas contemporâneas de intervenção coronária percutânea, a incidência de complicações vasculares foi de 6,5\%. Essa incidência está de acordo com os dados previamente publicados e relatados, demonstrando que, a despeito da melhoria da técnica e dos dispositivos, esse tipo de complicação permanece sendo relativamente freqüente $\mathrm{e}^{1-3}$. Por outro lado, também não se observou aumento da ocorrência dessas complicações como resultado do mais intenso regime de antiagregação plaquetária e da maior complexidade dos procedimentos realizados atualmente, em comparação com registros históricos publicados anteriormente $\mathrm{i}^{1-3}$.

O emprego dos inibidores da glicoproteína Ilb/IIla e o sexo feminino foram, neste estudo, apontados como preditores independentes de complicações vasculares, o que está de acordo com um estudo recente que uti- lizou as bases dos estudos Randomized Evaluation in PCl Linking Angiomax to Reduced Clinical Events 1 e 2 (REPLACE-1 e REPLACE-2) para criação e validação de um escore de risco para a ocorrência de complicações hemorrágicas após intervenção coronária percutânea ${ }^{15}$. Os inibidores da glicoproteína Ilb/IIla são potentes bloqueadores da agregação plaquetária e estudos publicados anteriormente também encontraram maiores taxas de complicações vasculares com seu emprego ${ }^{1,8,16,17}$. Em relação ao sexo feminino, os resultados desta investigação confirmam os achados do registro dinâmico do National Heart Lung and Blood Institute, dos Estados Unidos, com intervenções percutâneas realizadas entre 1997 e 1998, e, também, os dados de um estudo multicêntrico mais contemporâneo, publicado em 2004, com 63 mil mulheres submetidas a procedimentos diagnósticos e terapêuticos ${ }^{14,18}$. Neste último, as complicações vasculares foram duas vezes mais freqüentes no sexo feminino, em comparação com o sexo masculino ${ }^{14}$. Diversas outras publicações também corroboram esse achado1,3,8,15,17,19. Não está claro se essa maior propensão a complicações vasculares se deve apenas ao gênero feminino ou se pode ser explicada por outros fatores, como, por exemplo, menor superfície corpórea, maior sensibilidade aos anticoagulantes e antiplaquetários ou diferenças hormonais. Talvez o menor tamanho corporal e, conseqüentemente, o menor calibre das artérias no sexo feminino possam

\section{Odds ratio [IC 95\%]}

$5,61[1,99-15,76]$

$3,02[1,10-8,26]$

$0,36[0,12-0,99]$

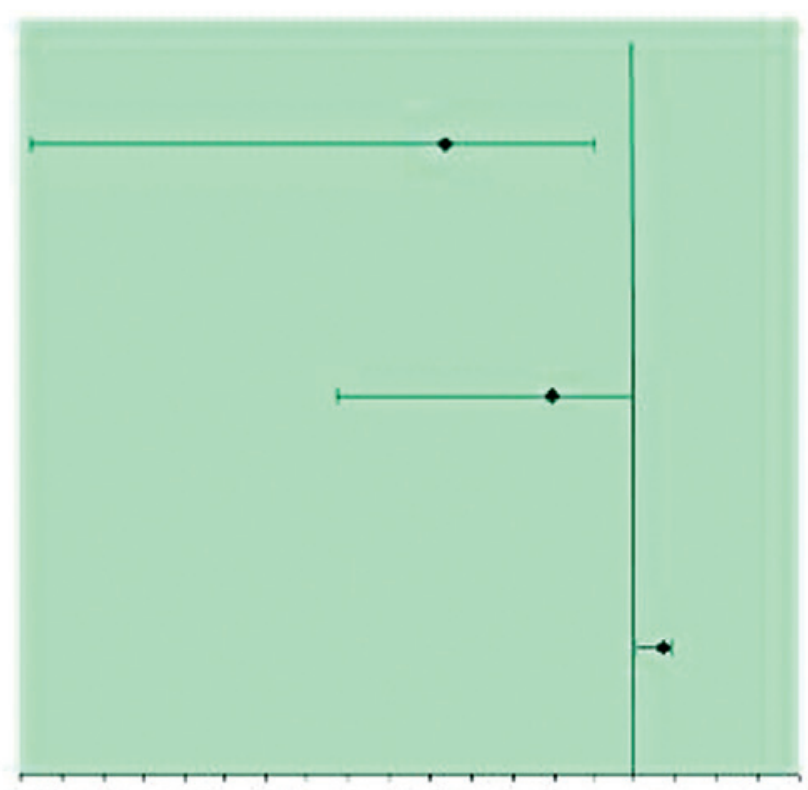

Sexo ferninino

Abciximab

Dispositivo hemostático

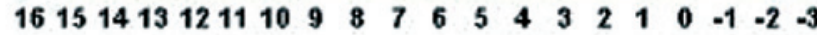

Figura 1 - Representação da análise multivariada com os preditores independentes de complicação vascular. O modelo final apresentou boa capacidade discriminatória para a presença de complicação vascular (área sob a curva = 0,76; IC 95\% = 0,69-0,84). A estatística de HosmerLemeshow goodness-of-fit não foi estatisticamente significante (8 df $=6,73 ; \mathrm{p}=0,565)$, demonstrando ajuste adequado do modelo com essa população. IC $95 \%$ = intervalo de confiança de $95 \%$. 
Brito Jr. FS, et al. Incidência e Preditores Contemporâneos de Complicações Vasculares após Intervenção Coronária Percutânea. Rev Bras Cardiol Invas 2007; 15(4): 394-399.

contribuir para a maior ocorrência das complicações vasculares oclusivas e isquêmicas nessa população, como sugerem outros autores ${ }^{14}$. A punção arterial também é, em geral, mais complexa em artérias de menor calibre, com maior propensão a múltiplas punções, transfixação da parede posterior do vaso e, conseqüentemente, hemorragias. Entretanto, na presente investigação, o índice de massa corporal não foi um preditor de complicações vasculares nas análises uni e multivariadas. Além disso, cabe destacar que, habitualmente, as doses de heparina são ajustadas pelo peso do paciente e, na presente investigação, os níveis de anticoagulação, avaliados pelo TCA, não foram diferentes nos sexos masculino e feminino. Outra possibilidade, ainda que especulativa, para explicar a maior ocorrência de complicações no sexo feminino pode estar relacionada à maior sensibilidade aos agentes antiplaquetários, como resultado de polimorfismos genéticos nos receptores das plaquetas ${ }^{17}$. Uma meta-análise de estudos com inibidores da glicoproteína IIb/IIla apóia essa hipótese, ao demonstrar maior ocorrência de complicações hemorrágicas em indivíduos do sexo feminino ${ }^{17}$. Adicionalmente, o estudo REPLACE-2, que avaliou o uso da bivalirudina em comparação com a associação de heparina não-fracionada e um inibidor da glicoproteína Ilb/IIla, demonstrou que as mulheres tiveram maiores benefícios que os homens com a bivalirudina ${ }^{20}$. Diante dessas informações e na ausência de uma explicação definitiva e consensual para a maior incidência de complicações vasculares no sexo feminino, julgamos que, antes do emprego dos inibidores da glicoproteína Ilb/IIla nessa população, os riscos de sangramento devem ser avaliados criteriosamente em relação a seus benefícios potenciais, reservando seu uso para situações em que as evidências de benefício, na visão do operador, sejam inequívocas. Cabe destacar que o emprego rotineiro no sexo feminino do esquema antitrombótico alternativo com bivalirudina, embora possa parecer atraente, está limitado, em nosso meio, pela indisponibilidade da droga e por seu custo elevado, em comparação à heparina não-fracionada. Obviamente, esse achado consistente de maior propensão para ocorrência de complicações vasculares no sexo feminino não deve ser, no nosso entendimento, motivo para restringir a indicação de procedimentos terapêuticos percutâneos nas mulheres, mas, sim, para estimular a investigação de estratégias alternativas, que possibilitem a realização dessas intervenções com a mesma segurança com que são feitas no sexo masculino.

Outro achado interessante desta investigação refere-se ao efeito protetor dos dispositivos hemostáticos, especialmente nos indivíduos do sexo masculino, e com o emprego de dispositivos hemostáticos do tipo plugue de colágeno, utilizado majoritariamente neste estudo. Os dispositivos hemostáticos, certamente, facilitam a recuperação e melhoram o conforto dos pacientes, que podem deambular mais precocemente ${ }^{11,21}$. Entretanto, os estudos que avaliaram seu impacto sobre a ocorrência de complicações vasculares têm resultados ambíguos ${ }^{4,9-14}$. Alguns demonstraram aumento da incidência dessas complicações, enquanto outros demonstraram efeito protetor ou nenhuma diferença em relação à tradicional técnica de compressão manual ${ }^{4,9-14}$. Há, ainda, estudos indicando que as mulheres têm maior risco de ocorrência de eventos adversos hemorrágicos associados ao uso dos dispositivos hemostáticos, o que não foi detectado nesta casuística ${ }^{22-24}$. Entretanto, é importante enfatizar que, no presente estudo, o emprego dos dispositivos hemostáticos e a escolha do tipo do dispositivo ocorreram de forma não-randomizada e dependeram unicamente da decisão do operador. Dessa forma, é possível que tenha ocorrido uma seleção de casos de menor complexidade para seu uso. Seguramente, casos que já apresentavam hematoma ou algum tipo de complicação no local da punção arterial, durante a intervenção, foram selecionados para compressão manual. Pacientes com doença vascular periférica acentuada ou aqueles em que a angiografia identificava punção arterial na bifurcação das artérias femorais também não receberam os dispositivos hemostáticos. Esse viés de seleção poderia explicar, pelo menos em parte, o aparente efeito protetor desses dispositivos, encontrado nesta investigação. Os autores reconhecem, ainda, que o presente estudo avaliou um número relativamente pequeno de pacientes e que, por esse motivo, a ampliação dessa casuística é fundamental para confirmar esses achados.

\section{REFERÊNCIAS BIBLIOGRÁFICAS}

1. Blankenship JC, Hellkamp AS, Aguirre FV, Demko SL, Topol EJ, Califf RM. Vascular access site complications after percutaneous coronary intervention with abciximab in the Evaluation of c7E3 for the Prevention of Ischemic Complications (EPIC) trial. Am J Cardiol. 1998;81(1):36-40.

2. Kinnaird TD, Stabile E, Mintz GS, Lee CW, Canos DA, Gevorkian N, et al. Incidence, predictors, and prognostic implications of bleeding and blood transfusion following percutaneous coronary interventions. Am J Cardiol. 2003;92(8): 930-5.

3. Piper WD, Malenka DJ, Ryan TJ Jr, Shubrooks SJ Jr, O'Connor GT, Robb JF, et al. Predicting vascular complications in percutaneous coronary interventions. Am Heart J. 2003;145(6): 1022-9.

4. Applegate RJ, Sacrinty MT, Kutcher MA, Baki TT, Gandhi SK, Santos RM, et al. Propensity score analysis of vascular complications after diagnostic cardiac catheterization and percutaneous coronary intervention 1998-2003. Catheter Cardiovasc Interv. 2006;67(4):556-62.

5. Lauer MA, Karweit JA, Cascade EF, Lin ND, Topol EJ. Practice patterns and outcomes of percutaneous coronary interventions in the United States: 1995 to 1997. Am J Cardiol. 2002;89(8):924-9.

6. Kugelmass AD, Cohen DJ, Brown PP, Simon AW, Becker ER, Culler SD. Hospital resources consumed in treating complications associated with percutaneous coronary interventions. Am J Cardiol. 2006;97(3):322-7.

7. Kuchulakanti PK, Satler LF, Suddath WO, Pichard AD, Kent KM, Pakala R, et al. Vascular complications following coronary intervention correlate with long-term cardiac events. Catheter Cardiovasc Interv. 2004;62(2):181-5. 
Brito Jr. FS, et al. Incidência e Preditores Contemporâneos de Complicações Vasculares após Intervenção Coronária Percutânea. Rev Bras Cardiol Invas 2007; 15(4): 394-399.

8. Yatskar L, Selzer F, Feit F, Cohen HA, Jacobs AK, Williams DO, et al. Access site hematoma requiring blood transfusion predicts mortality in patients undergoing percutaneous coronary intervention: data from the National Heart, Lung, and Blood Institute Dynamic Registry. Catheter Cardiovasc Interv. 2007;69(7):961-6.

9. Nikolsky E, Mehran R, Halkin A, Aymong ED, Mintz GS, Lasic Z, et al. Vascular complications associated with arteriotomy closure devices in patients undergoing percutaneous coronary procedures: a meta-analysis. J Am Coll Cardiol. 2004;44(6):1200-9.

10. Dangas G, Mehran R, Kokolis S, Feldman D, Satler LF, Pichard AD, et al. Vascular complications after percutaneous coronary interventions following hemostasis with manual compression versus arteriotomy closure devices. J Am Coll Cardiol. 2001;38(3):638-41.

11. Silber S. Rapid hemostasis of arterial puncture sites with collagen in patients undergoing diagnostic and interventional cardiac catheterization. Clin Cardiol. 1997;20(12):981-92.

12. Arora N, Matheny ME, Sepke C, Resnic FS. A propensity analysis of the risk of vascular complications after cardiac catheterization procedures with the use of vascular closure devices. Am Heart J. 2007;153(4):606-11.

13. Exaire JE, Tcheng JE, Kereiakes DJ, Kleiman NS, Applegate RJ, Moliterno DJ. Closure devices and vascular complications among percutaneous coronary intervention patients receiving enoxaparin, glycoprotein $\mathrm{Ilb} / \mathrm{Illa}$ inhibitors, and clopidogrel. Catheter Cardiovasc Interv. 2005;64(3):369-72.

14. Tavris DR, Gallauresi BA, Lin B, Rich SE, Shaw RE, Weintraub WS, et al. Risk of local adverse events following cardiac catheterization by hemostasis device use and gender. J Invasive Cardiol. 2004;16(9):459-64.

15. Nikolsky E, Mehran R, Dangas G, Fahy M, Na Y, Pocock $S$ J, et al. Development and validation of a prognostic risk score for major bleeding in patients undergoing percutaneous coronary intervention via the femoral approach. Eur Heart J. 2007;28(16):1936-45.

16. ESPRIT Investigators. Enhanced Suppression of the Platelet Ilb/Illa Receptor with Integrilin Therapy. Novel dosing regimen of eptifibatide in planned coronary stent implantation
(ESPRIT): a randomised, placebo-controlled trial. Lancet. 2000;356(9247):2037-44.

17. Boersma E, Harrington RA, Moliterno DJ, White $H$, Theroux $\mathrm{P}$, Van de Werf $\mathrm{F}$, et al. Platelet glycoprotein Ilb/Illa inhibitors in acute coronary syndromes: a meta-analysis of all major randomised clinical trials. Lancet. 2002;359(9302):189-98.

18. Jacobs AK, Johnston JM, Haviland A, Brooks MM, Kelsey SF, Holmes DR Jr, et al. Improved outcomes for women undergoing contemporary percutaneous coronary intervention: a report from the National Heart, Lung, and Blood Institute Dynamic registry. J Am Coll Cardiol. 2002;39(10):1608-14.

19. Farouque HM, Tremmel JA, Raissi Shabari F, Aggarwal M, Fearon WF, Ng MK, et al. Risk factors for the development of retroperitoneal hematoma after percutaneous coronary intervention in the era of glycoprotein Ilb/llla inhibitors and vascular closure devices. J Am Coll Cardiol. 2005;45(3):363-8.

20. Lincoff AM, Bittl JA, Harrington RA, Feit F, Kleiman NS, Jackman JD, et al. Bivalirudin and provisional glycoprotein $\mathrm{Ilb} / \mathrm{Illa}$ blockade compared with heparin and planned glycoprotein $\mathrm{Ilb} / \mathrm{Ill}$ a blockade during percutaneous coronary intervention: REPLACE-2 randomized trial. JAMA. 2003;289 (7):853-63

21. Duffin DC, Muhlestein JB, Allisson SB, Horne BD, Fowles $\mathrm{RE}$, Sorensen $\mathrm{SG}$, et al. Femoral arterial puncture management after percutaneous coronary procedures: a comparison of clinical outcomes and patient satisfaction between manual compression and two different vascular closure devices. Invasive Cardiol. 2001;13(5):354-62.

22. Carere RG, Webb JG, Miyagishima R, Djurdev O, Ahmed T, Dodek A. Groin complications associated with collagen plug closure of femoral arterial puncture sites in anticoagulated patients. Cathet Cardiovasc Diagn. 1998;43(2):124-9.

23. Sesana $M$, Vaghetti $M$, Albiero R, Corvaja N, Martini G, Sivieri G, et al. Effectiveness and complications of vascular access closure devices after interventional procedures. J Invasive Cardiol. 2000;12(8):395-9.

24. Tavris DR, Gallauresi B, Rich S, Bell C. Relative risks of reported serious injury and death associated with hemostasis devices by gender. Pharmacoepidemiol Drug Saf. 2003;12(3): $237-41$ 